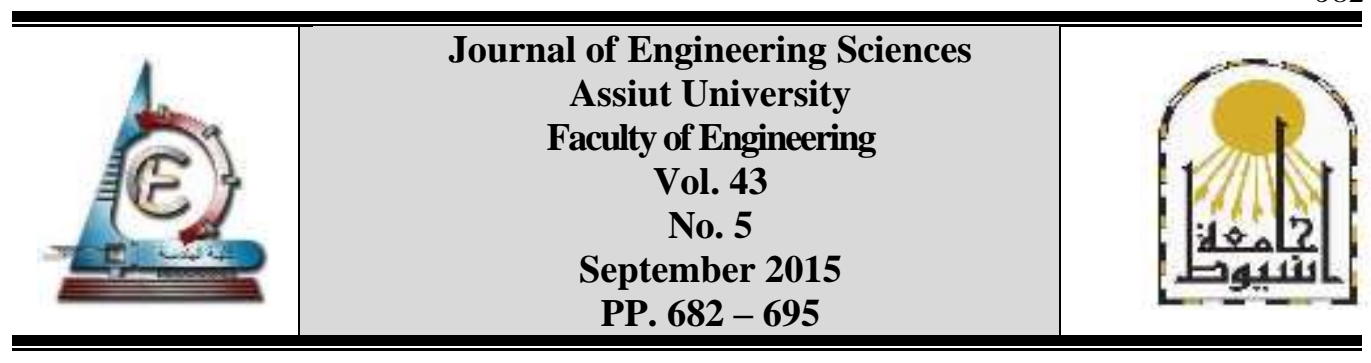

\title{
AUTONOMAS FAULT DIAGNOSIS SYSTEM FOR CELLULAR NETWORKS BASED ON HIDDEN MARKOV MODEL
}

\author{
Omar AbdelMoez ", Asem M. Ali, T.K. Abdelhamid \\ Faculty of Engineering, Assiut University, Assiut, Egypt
}

(Received 21 April 2015; Revised 9 May 2015; Accepted 27 August 2015)

\begin{abstract}
Automated diagnosis and Troubleshooting (TS) in Radio Access Networks (RAN) of cellular systems are basic management tasks, which are required to guarantee efficient use of network resources. In this paper, we investigate the usage of machine learning techniques: stochastic methods and discriminant analysis for automating these TS tasks. Our proposed framework is based on Hidden Markov Model (HMM), Principle Component Analysis (PCA) and Fisher Linear Discriminant (FLD) techniques. In a learning phase, symptoms relating to faults in the network are extracted from a network management system (NMS). Then they are used to create a fault model. This model is used to identify the unknown faults using a nearest neighbor classifier. Reported results for the automated diagnosis using live RAN measurements illustrate the efficiency of the proposed TS framework and its importance to mobile network operators.
\end{abstract}

Keywords: Automated diagnosis, Hidden Markov Model (HMMs), faults, symptoms, troubleshooting (TS), the Next Generation Mobile Networks (NGMN).

\section{Introduction}

Mobile services are growing faster than most industry observers' predictions. Market demands and competition are driving the needs for a rapid expansion in network capacity, coverage, and quality to meet rising user expectations in terms of connection speed and network availability. This leads to huge and complex networks with thousands of cells. In the meanwhile, operators of mobile communication networks face the challenging task of managing such complex networks.

Along with the Long Term Evolution (LTE) introduction, the concept of Self Organizing Networks (SON) [1] was adopted by the Next Generation Mobile Networks (NGMN) alliance to address challenges of management several radio access technologies. SON categorized into functional tasks according to the operation and maintenance areas of configuration, optimization and troubleshooting. Accordingly, SON includes self-configuration, self-optimization and selfhealing network features (shown in Fig.1). Self healing function aims to automate fault detection, diagnosis and recovery [2], especially, for coverage and capacity problems to compensate

\footnotetext{
* Corresponding author.

Email address: omar.abdelmoez@gmail.com
} 
network outages and enhance coverage performance. In practice, operation teams perform fault recovery either through remote action like a remote reset for hardware to recover hanging or through field intervention to fix the defected item. Most of SON proposed frameworks (e.g., [1][2]), concerned with self healing through remote fault recovery and in case of failure of the remote recovery. SON tries to change the configuration management $(\mathrm{CM})$ parameters for network elements [2] to provide a solution for the radio coverage problems. As an example, to compensate the coverage gap of the impacted neighbour cell, the base station power is increased. Despite of the high time consumption needed to make alarms and Key Performance Indicators (KPIs) correlations to diagnose the second type of faults, which need field intervention, this type of diagnosis has not been addressed under SON standardization [1]. In this paper, automatic fault diagnosis addresses the latter type of faults, more specifically the radio base station hardware and transmission network faults, to facilitate complex network troubleshooting tasks.

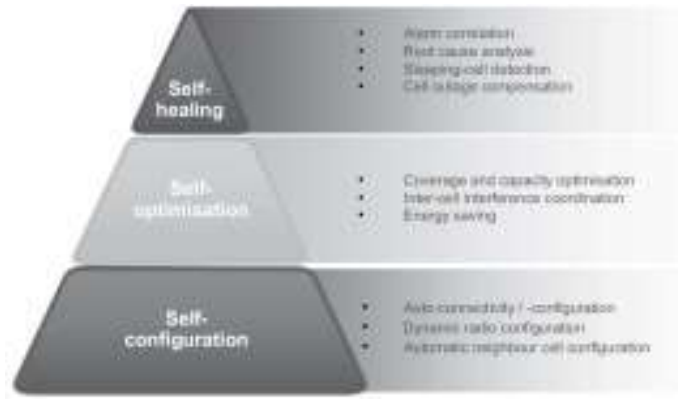

Fig. 1. SON Use Case Examples [1].

Mobile networks are divided into two main parts, the core network and the access network. The access network is the part of a telecommunications network that connects subscribers to their immediate service provider [3]. While the core network is the central part of any telecommunication network that provides various services to customers, who are connected by the access network. One of the core network's main functions is to route telephone calls to other mobile operators and the Public Switched Telephone Network (PSTN) [4].

In the second generation mobile networks (GSM), the access network consists of three main parts, a radio base transceiver station (BTS), the base station controller (BSC), and transmission links connecting the base station to the base station controller. In the third generation mobile networks (UMTS) the radio access

The network consists of three main parts also, a radio base station, which is called a NodeB, the radio network controller (RNC), and transmission links, which connecting RNC to NodeB. While in the Long Term Evolution mobile networks (LTE), the access network consists of multiple of enhanced NodeB (eNodeB) and transmission links connecting eNodeBs (eNB) with each other and to the core network.

Modern mobile networks support multiple radio technologies of GSM, UMTS, LTE mobile networks and WIFI network. The transmission links can be microwave link, optical fiber, copper lines, laser optics link or satellite link.

Different transmission links are interconnected together to provide different access network connectivity forming a private transmission network for mobile operators as depicted in Fig.2. 


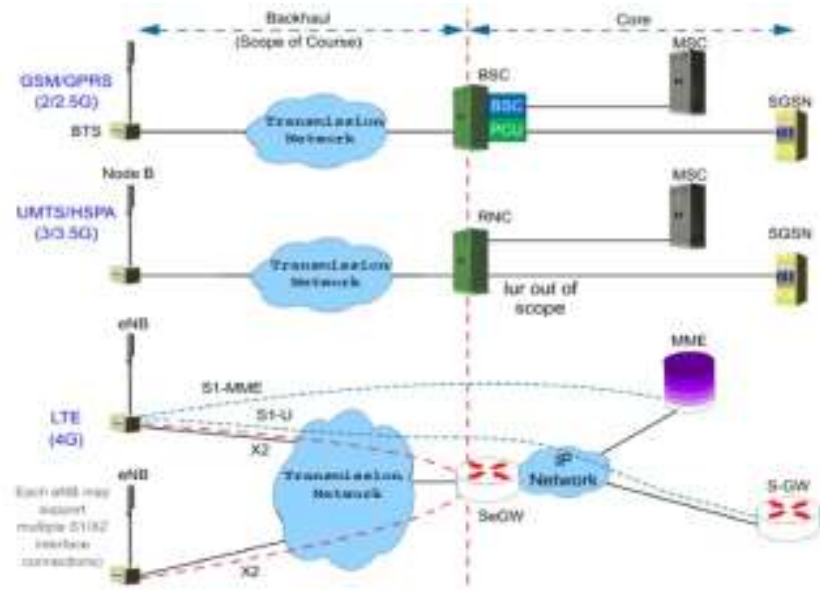

Fig. 2. Multiple Radio Access Technologies Mobile Network.

Tools are required to automatically analyze the data that are collected from different parts of the network. This provides a useful feedback that can be smartly used for mobile business priorities, managing network complexity, save revenue loss and improves customer experience.

Manage access networks need a large number of operation experts, who diagnose network's faults as well as propose quick solutions for restoring the network. There are two kinds of operation experts involved in radio access network faults' troubleshooting. Radio optimization experts [5] perform radio coverage faults diagnosis and recovery. Radio Access Network (RAN) operation and maintenance experts [6] perform maintenance for hardware of radio access network nodes BTS and transmission links.

The main symptoms, which can be adopted in identifying RAN's faults, are Key Performance Indicators (KPIs) and alarms. Examples of KPIs [7] are Blocked Call Rate (BCR), Dropped Call Rate (DCR), network availability, etc. While base station modules' faults, which composed of elements that deteriorate gradually or suddenly over time, generate alarms. The faulty component can be a transceiver (TRX), a radio combiner, an antenna, a connector, a cable, a power supply, etc.

\section{Related work}

Automating TS consolidates thousands of unlabelled alarms and KPIs into meaningful events, which can be easily managed. Still the research in the area of automatic diagnosis in the radio access part of cellular networks is limited, despite of the increasing interest in this type of research shown by mobile network operators and manufacturers. Among troubleshooting tasks, diagnosing the cause of faults is the most complex and timeconsuming one. Therefore, researchers investigated the automatic diagnoses of different faults. As an example, Barco et al. [8] focused on BTS hardware faults, e.g., faulty TRX. While in another work, Baku et al. [9] focused on transmission link faults. Coverage faults, e.g., lack of coverage, were investigated in ([10] - [11]).

In the limited previous works ([8] - [11]) KPIs and alarm symptoms were used in discrete Bayesian networks-based frameworks for automatic fault diagnosis in cellular networks. Solana et al. [11] used KPIs as symptoms for radio coverage faults diagnosis. While Barco et al. [9] used a KPI and alarms as symptoms to diagnose hardware and radio coverage faults. However, low diagnosis accuracy has been achieved as only dropped call 
rate (DCR) KPI adopted as symptoms. On the other hand, in other works, e.g., ([10], [11]) KPIs only are used as symptoms to diagnose radio coverage faults, hardware faults and transmission link faults. However, low diagnosis accuracy achieved for hardware and transmission link faults as alarms have not been considered as symptoms.

Previous automated fault diagnosis researches in mobile network focus on radio coverage faults and its self-healing mechanism as an important part of a self-organizing network (SON) architecture [7]. Radio KPIs as symptoms represent a strong information source to reveal radio coverage faults. Despite of the importance and complexity of hardware and transmission link faults and its significant contribution in service outages, hardware and transmission faults are not efficiently addressed in these works e.g., ([10] -[11]).

In this work, hardware and transmission link faults are deeply investigated to facilitate the most consuming time in troubleshooting complex hardware and transmission faults come from multi radio access technology networks shown in Fig.2. Since alarms are considered as rich information sources to identify hardware and transmission link faults, alarms have been selected as symptoms. Our main objective is designing a tool to automatically achieve high diagnostic accuracy and to be easily implemented in an operator live network. In this paper, Hidden Markov Model (HHM) ([12]-[14]) is adopted for diagnosis. HMM is used as a statistical method for modelling and recognizing sequential information (i.e. Alarms consequences).

\section{Proposed framework}

The access network, which is addressed in this paper, consists of more than 6000 of physical radio base stations. These base stations are connected to base station controllers and radio network controllers using more than 6000 different types of links. Links covered in this paper are mainly fixed microwave links. Microwave links use the free space as a transmission medium and a beam of radio waves in the microwave frequency range to transmit data between two locations. The microwave link consists of two main parts: an indoor unit, which performs baseband digital signal processing, and an outdoor unit, which performs radio signal processing. In this paper, the microwave fault of the baseband and the radio units has been addressed.

On the other hand, the base transceiver station consists of three main parts: a clock card to interface with the BSC and to perform the baseband processing, a transceiver to perform most of the radio wave processing, and an antenna system to convey the radio waves from the transceiver to be transmitted to mobile stations. In this paper, two types of antenna system faults are addressed.

These four types of faults are chosen because they contribute significantly in the overall mobile network faults. Since the target access network is a complex and a high dimensional network, this network uses microwave links which suffer from many radio propagation problems, i.e., interference, fading... etc.

In the proposed framework, alarms as symptoms are gathered from network management system NMS [20]. The target radio access network NMS reports many categories indicating BTS faults namely: hardware, software, quality of service, signalling, trunk, and communication faults. In this work, BTS communication trunk faults (i.e. The microwave fault of the baseband and the radio units) and GSM antenna faults (two types of antenna system faults) have been addressed. For each fault type, alarms are daily recorded in a historical file. 


\subsection{HMM-Based modelling and recognition}

Because it has been successfully used for automatic diagnosis in many applications (e.g., diagnosis of diseases in medicine [15-17] and diagnosis of mechanical system faults [18-19]), we propose to use Hidden Markov Model (HMM) [12] in diagnosing faults of multi radio access technologies networks. The alarmed data, in each historical file, are segmented into T segments; each segment has a duration of $\mathrm{K}$ seconds. Then each segment is sampled every $\tau$ seconds. Finally, each alarm message is given an arbitrary code. The result is an observations matrix $\mathrm{O}=\left\{\mathrm{O}_{1}, \mathrm{O}_{2}, \mathrm{O}_{3}, \ldots, \mathrm{O}_{\mathrm{T}}\right\}$ of $\mathrm{K} / \tau$ codes for each observation vector $\mathrm{O}_{\mathrm{i}},(1 \leq \mathrm{i} \leq \mathrm{T})$ and $\mathrm{T}$ varies based on the alarm historical file length.
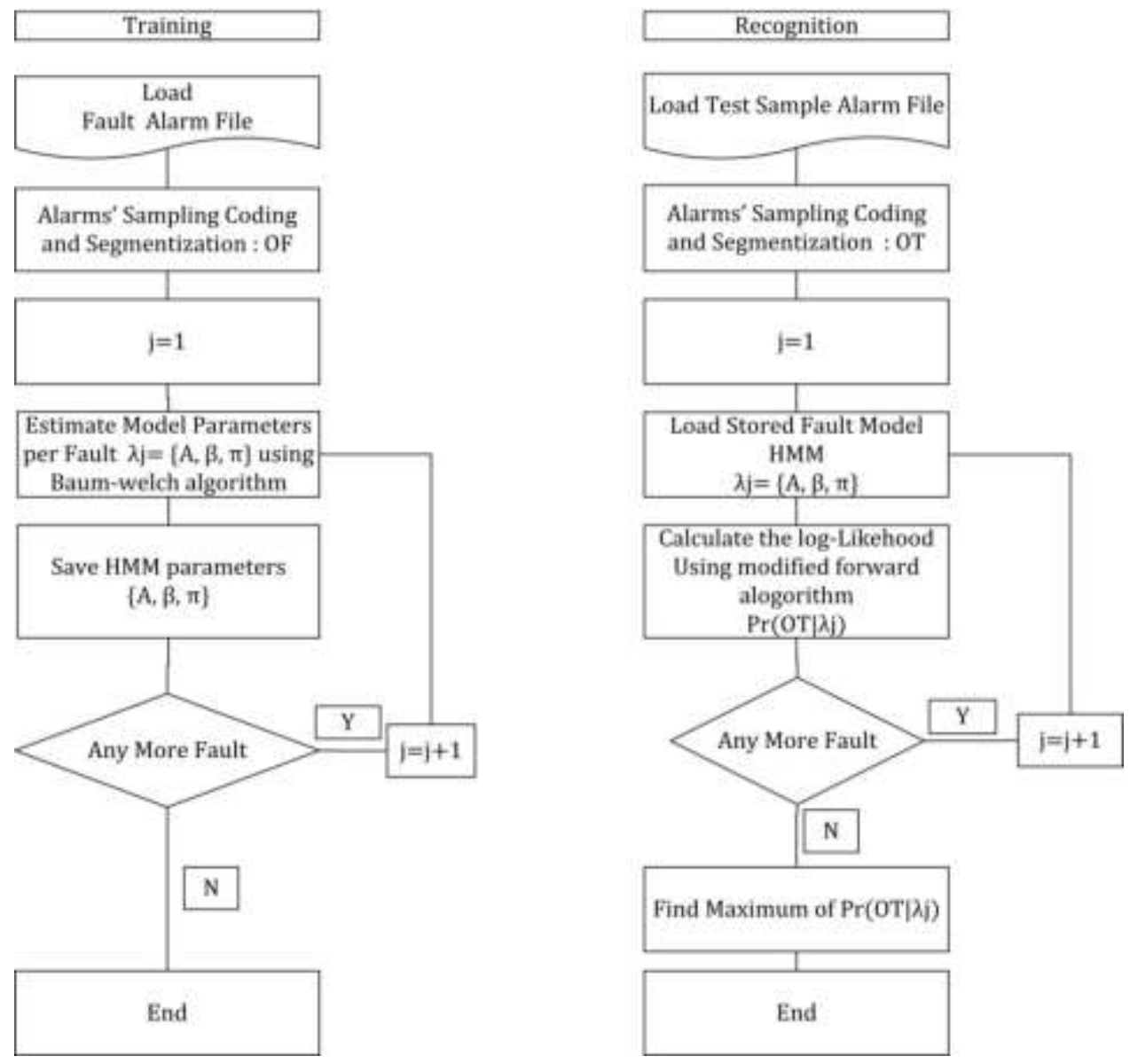

Fig. 3. HMM Training and Recognition Approach.

As shown in Fig. 3, in the training phase the faults' observation sequences $\mathrm{O}_{\mathrm{F}}$ (i.e. Coded alarms) are used in modelling and training for each fault model $\lambda_{\mathrm{j}}$ using Baum-Welch's algorithm [12]. While in the recognition phase, an under test fault's observation sequence (i.e. Coded alarms) is used to calculate the likelihood $\operatorname{Pr}\left(O_{T} \mid \lambda_{j}\right)$ of the under test faults' observation $\mathrm{O}_{\mathrm{T}}$ with respect to each fault model $\lambda_{\mathrm{j}}$ using the modified forward algorithm.

HMM is useful to model a sequential event in a system [21]. In this work, HMM is used as a statistical method for modelling and recognizing sequential information (i.e. Coded alarm consequences). The purpose of training a HMM is to determine a model parameters 
$\lambda_{j}$ with the highest probability of the observation likelihood $\operatorname{Pr}\left(O_{F} \mid \lambda_{j}\right)$ when observing a sequential alarm's code under test $\mathrm{O}_{\mathrm{F}}$.

For a set of hidden states $S=\left\{s_{1}, s_{2} \ldots \ldots s_{N}\right\}$, the conventional HMM model is denoted as $\lambda=\{\mathrm{A}, \mathrm{B}, \pi\}$. Where the state transition probability matrix $\mathrm{A}$, the observation symbol probability matrix $\mathrm{B}$, and the initial state $\pi$ are defined as

$$
\begin{array}{lll}
A=\left\{a_{i l}\right\}, & a_{i l}=P_{r}\left(q_{t+1}=S_{l} \mid q_{t}=S_{i}\right), 1 \leq i, l \leq N \\
B=\left\{b_{l}\left(O_{t}\right)\right\}, & b_{l}=P_{r}\left(O_{t} \mid q_{t}=S_{l}\right), 1 \leq l \leq N \\
B=\left\{b_{l}\left(O_{t}\right)\right\}, & b_{l}=P_{r}\left(O_{t} \mid q_{t}=S_{l}\right), 1 \leq l \leq N \\
\pi=\left\{\pi_{l}\right\}, & \pi_{l}=P_{r}\left(q_{1}=S_{l}\right)
\end{array}
$$

In this work, a hidden state $s_{i}$ represents the unique signature for a fault when HMM trained using the alarm sequence $\mathrm{O}_{\mathrm{F}}$. In the learning phase of the proposed framework, we define the variable, $\xi(i, l)$ as the probability of being in the state $s_{i}$ at time $t$, and the sate $s_{1}$ at time $t+1$, and the variable $\gamma(\mathrm{i})$, as the probability of being in the state $s_{1}$ at time $t$ as follows.

$$
\begin{gathered}
\xi(i, l)=\frac{\alpha_{t} a_{i l} b_{l}\left(O_{t+1}\right) \beta_{t+1}(l)}{\operatorname{Pr}(O \mid \lambda)} \\
\gamma_{t}(i)=\sum_{l=1}^{N} \xi(i, l)
\end{gathered}
$$

Also define $\alpha_{\mathrm{t}}(\mathrm{i})$ as the forward variable and $\beta_{\mathrm{t}}(\mathrm{i})$ as the backward variable:

$$
\begin{array}{lc}
\alpha_{t}(i)=\pi_{i} b_{i}\left(O_{1}\right), & (1 \leq i \leq q) \\
\alpha_{t+1}(l)=\left[\sum \alpha_{t}(i) a_{i l}\right] b_{l}\left(O_{t+1}\right), & (t=1,2, \ldots \ldots, T-1) \\
\beta_{t}(i)=1, & (1 \leq i \leq q) \\
\beta_{t}(i)=\sum_{j=1}^{N} a_{i j} b_{j}\left(O_{t+1}\right) \beta_{t+1}(i), & (t=T-1, T-2, \ldots \ldots, 1)
\end{array}
$$

Using these variables, we can estimate the updated parameters A and B of the model of $\lambda$ via estimating probabilities as follows.

$$
\begin{aligned}
& \overline{a_{l l}}=\frac{\sum_{t=1}^{T-1} \xi_{t}(i, l)}{\sum_{t=1}^{T-1} \gamma_{t}(i)} . \\
& \overline{b_{l}(k)}=\frac{\sum_{t=1}^{T-1} O_{t}=k}{\sum_{t=1}^{T-1} \gamma_{t}(i)} .
\end{aligned}
$$

Where $\overline{\mathrm{a}_{11}}$ is the estimated transition probability from the state $i$ to the state $l$ and $\overline{b_{l}(k)}$ is the estimated observation probability of alarm's code $k$ from the statel. In the recognition phase of the proposed framework, the probability of a certain coded alarm $\mathrm{O}_{\mathrm{t}}$ generated from a fault model $\lambda_{\mathrm{j}}$ is calculated using the forward algorithm as follows. 
$\operatorname{Pr}\left(\mathrm{O}_{t} \mid \lambda_{j}\right)=\sum_{i=1}^{N} \alpha_{T}(i)$,

$\alpha_{\mathrm{t}}(\mathrm{i})$ is the probability of observations being in state $i$ at time $t$.

\subsection{Features selection and dimensionality reduction}

As mentioned before, alarms are daily recorded in a historical file, and then data are segmented and sampled. The dimension of the resultant fault signature may be greater than 7000 due to the high dimensionality of the alarm log file especially for a number of records, which spread over an extended time duration. It is well known that a large number of features may cause over-fitting, if they are not relevant features and if the underlying distributions are not estimated accurately. Furthermore, a large number of features make it difficult to design a classifier due to time and space complexity issues.

Therefore, in this work, we use two mechanisms to select the features. The first mechanism represents the original information in a lower dimensional space by retaining most of the relevant information. This is the Principal Component Analysis (PCA) approach [22]. The second mechanism, which is Fisher Linear Discriminant (FLD) [23], maps the original feature space into one where classes separability is greatly enhanced.

Principal Component Analysis (PCA) is used as a first technique followed by HMM. PCA linearly transforms fault data (i.e. Alarms) in the high-dimensional space to a space of fewer dimensions. Since HMM is normally trained with symbols of sequential data, the feature vectors obtained from PCA are quantized to constitute a codebook, which is a set of quantized signature of faults' sequential dataset. Then, the codebook is used in training HMM's, as explained in the flowchart shown in Fig. 5.

The PCA purpose is to find a linear orthogonal transformation $\mathrm{W}$ such that the variance is maximized in the new space (i.e., $v=W u, u$ is the observation vector.) In this work, $u$ represents the fault's raw data and $v$ represents the fault's feature vector. Alternatively, PCA represented a minimization of reconstruction error it turned out that these principles leads to a symmetric eigenvalue problem. The row vectors of $\mathrm{W}$ correspond to the normalized orthogonal eigenvectors of the data covariance matrix. Eigenvectors are obtained using singular value decomposition (SVD).

After training each HMM, an unknown fault test sequence is projected on the PCA feature space and quantized. The obtained symbols are compared with the codebook to form a proper test symbol set $\mathrm{O}$ to calculate the log-likelihood with respect to trained faults' models $\operatorname{Pr}\left(\mathrm{O} \mid \lambda_{\mathrm{j}}\right), 1 \leq \mathrm{j} \leq \mathrm{N}, \mathrm{N}$ is the number of trained faults.

PCA finds the minimum number of components that best represents the data. However, we need to reduce the dimensionality, under some constraint of maximizing the class discrimination. Therefore, we use Fisher Linear Discriminant (FLD) to map the original data space into another feature space that maximizes the class discrimination. Similar to PCA-based approach, the feature vectors obtained from FLD are quantized to constitute a codebook, then, the codebook is used in training HMM's.

Fig. 4. Presents an example of a codebook generation for four different faults, in the training phase the original data per fault is projected on the PCA feature space. The resultant faults' projection vectors are $f_{p 1}, f_{p 2}, f_{p 3}$ and $f_{p 4}$. After quantization, under the assumption 
of 1 quantized level per fault, there are four quantized levels $c_{1}, c_{2}, c_{3}$ and $c_{4}$ represent the codebook records and used to train the HMM. In the recognition phase, the test sample is projected on the PCA feature space, quantized according to the same quantization process used in the training phase and finally, HMM is used to find the highest likelihood of test sample's projection and codebook records as a codebook lookup process, as shown in Fig. 5.

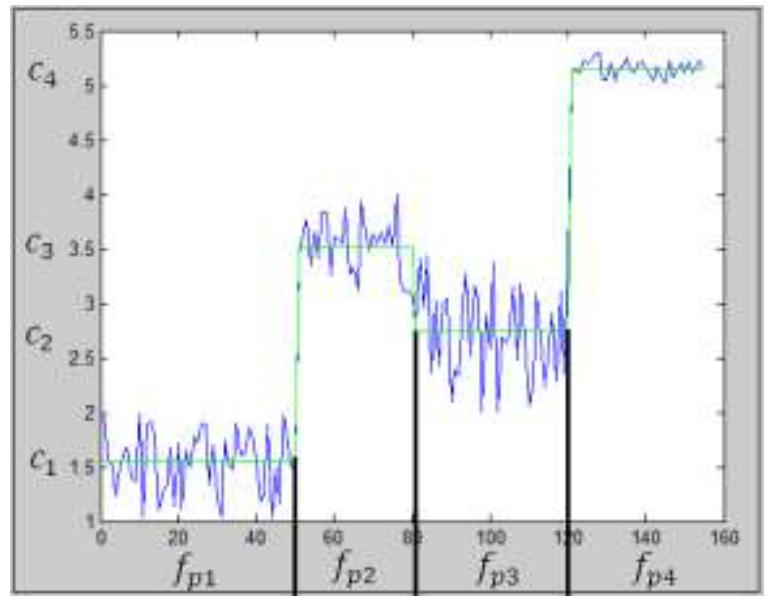

Fig. 4. PCA codebook Generation Example.

\section{Experiments}

To evaluate the performance of the proposed fault recognition system, a set of experiments is conducted for modelling and recognition. Also, the effect of feature dimensionality on the recognition performance is investigated. Where, machine learning approaches, PCA and FLD, are used in combination with HMM for modelling and recognition.

Experimental data has been collected from NMS of Vodafone Egypt live mobile network, alarms log files are gathered for three months duration. Four different types of faults have

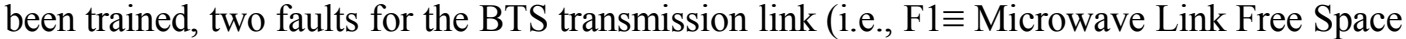
Propagation \& Transmission Fault and F2 $=$ Microwave Link hardware and Baseband Fault). Other two types are BTS antenna system faults (i.e., F3 $\equiv$ BTS Voltage Standing Wave Ratio (VSWR) [24] fault and F4三 Losing of Receiver Diversity Channel fault [25]). Our data consist of 74 alarm log fault files, at least 15 files per fault type. One file for each type is randomly selected for the training process and the others are used for testing.

To obtain accurate statistical results, the training and testing process are repeated many times using different files, and the average of the recognition performance is reported.

The reported results, in each experiment, represent the best recognition rate with the empirical settings of the raw observation vector length, number of features and codebook size. For PCA, eigenvectors have been computed for all faults datasets and the projections corresponding to the highest eigenvector are quantized and then used to train the HMM.

The system is quantitatively evaluated as follows. Each given test sample is identified against the four stored faults' models to get the recognition rate per fault. The recognition results are represented in the form of a confusion matrix, which represents the recognition rate for known test sample with respect to the fault models (i.e.,. For optimum classifier the confusion matrix is the identity matrix). Accordingly, the diagonal elements should have maximum values for each corresponding row. 
Omar abdelmoez et al., Autonomas fault diagnosis system for cellular networks based on hidden ....
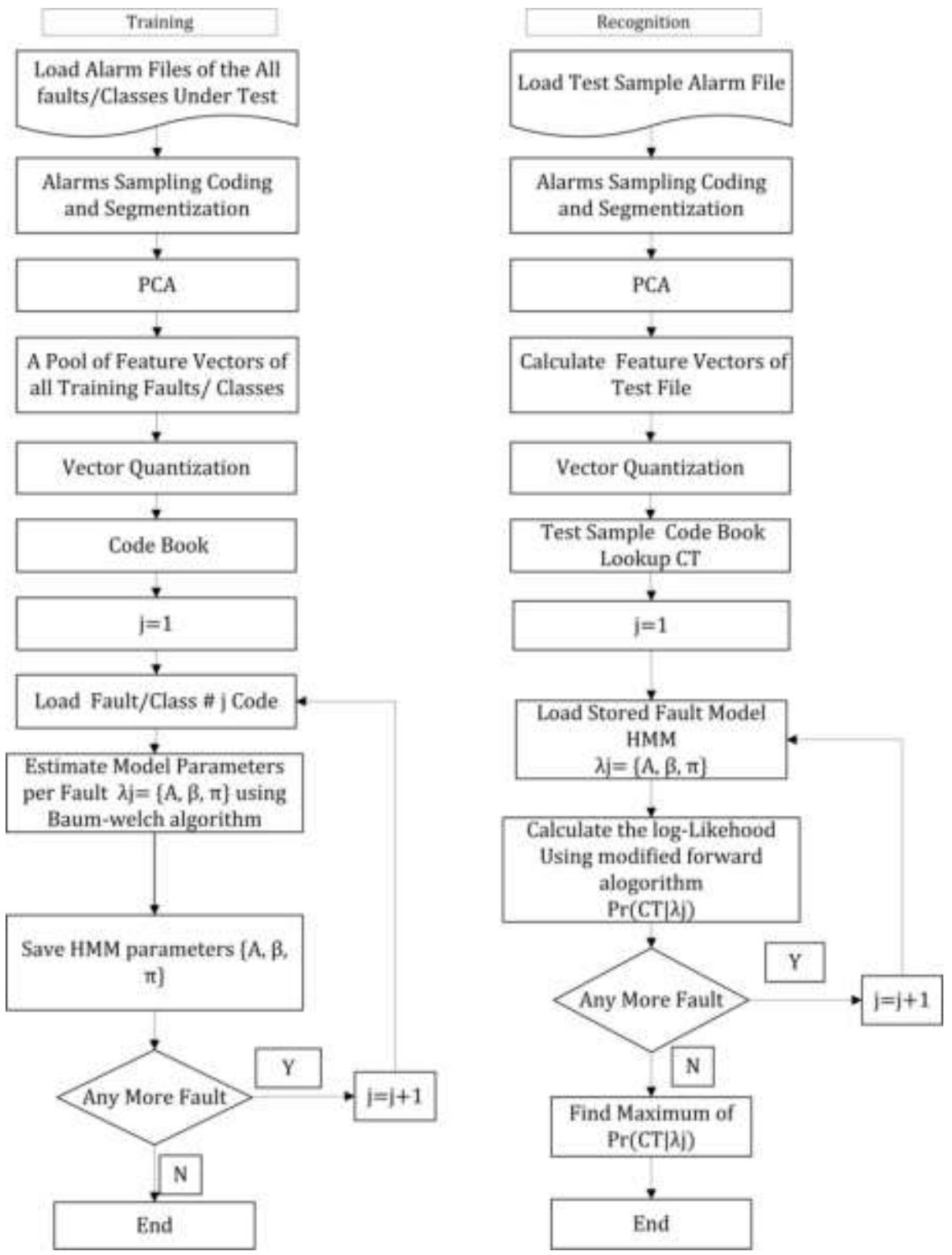

Fig. 5. A flowchart describes feature selection, training Procedure in the Left Side, and Recognition Procedures in the Right Side.

In the first experiment, the original data, which are segmented, sampled, and coded from the raw data, are used without any learning approach. A nearest neighbour classifier is used to classify the original data using the Euclidean distance. Table. 1 illustrates the recognition performance obtained from this experiment.

As expected, the original data, without learning, can't be correctly classified using a nearest neighbour classifier. This why fault F2 is identified as fault F1 and fault F4 is identified as fault F3. 
The second experiment addresses the usage of HMM in the recognition framework. After training the HMM's, the highest likelihood value for the unknown fault against each model is chosen as a criterion for the recognition. Table.2 illustrates the recognition performance obtained from this experiment. It is clear that the recognition performance is enhanced after using trained HMM's and the faults are correctly classified with an average recognition rate $78 \%$.

The third experiment addresses the usage of PCA in the recognition framework. The original data segmented, sampled, and coded, then PCA is applied to reduce the dimensions of coded data. A nearest neighbour classifier is used to classify the low dimensional PCA projected data using the Euclidean distance. Table. 3 illustrates the recognition performance obtained from this experiment. Again, fault F2 is identified as fault F1 and fault F4 is identified as fault F3. The results confirm that even the projected data cannot be classified without learning.

The fourth experiment addresses the usage of PCA or FLD in combination with HMM in the recognition framework. The original data segmented, sampled, and coded, then PCA or FLD is applied to reduce the coded data dimensions. HMM uses PCA codebook projected data to train faults. Then the highest likelihood value is used for recognition. Table.4 illustrates the recognition performance obtained by using PCA and HMM. Since the results of using FLD instead of PCA are comparable to the one in Table.4, we don't add these results. Despite the faults are correctly identified, the recognition performance is degraded compared to results reported in Table.2. This is due to information loss during the PCA codebook quantization process.

Table 1.

Original Data Euclidean Based Fault Diagnosis Confusion Matrix.

\begin{tabular}{|c|c|c|c|c|}
\hline Label & F1 & F2 & F3 & F4 \\
\hline F1 & $\mathbf{8 6 . 8 1}$ & 13.13 & 0.05 & 0.01 \\
\hline F2 & $\mathbf{6 0 . 7 2}$ & 39.08 & 0.20 & 0.00 \\
\hline F3 & 21.79 & 0.00 & $\mathbf{6 3 . 3 7}$ & 14.84 \\
\hline F4 & 30.02 & 0.00 & $\mathbf{6 0 . 6 6}$ & 9.32 \\
\hline Average & \multicolumn{4}{|c}{$49.64 \%$} \\
\hline
\end{tabular}

Table 2.

HMM Based Fault Diagnosis Confusion Matrix.

\begin{tabular}{|c|c|c|c|c|}
\hline Label & F1 & F2 & F3 & F4 \\
\hline F1 & $\mathbf{7 4 . 2 0}$ & 17.43 & 8.37 & 0.00 \\
\hline F2 & 28.13 & $\mathbf{7 1 . 4 3}$ & 0.44 & 0.00 \\
\hline F3 & 0.00 & 0.00 & $\mathbf{9 5 . 5 6}$ & 4.44 \\
\hline F4 & 0.03 & 0.00 & 29.25 & $\mathbf{7 0 . 7 2}$ \\
\hline Average & \multicolumn{5}{|c}{$77.98 \%$} \\
\hline
\end{tabular}


Table 3.

Original Data PCA Based Fault Diagnosis Confusion Matrix.

\begin{tabular}{|c|c|c|c|c|}
\hline Label & F1 & F2 & F3 & F4 \\
\hline F1 & $\mathbf{8 1 . 2 2}$ & 12.88 & 0.36 & 5.53 \\
\hline F2 & $\mathbf{6 3 . 6 2}$ & 33.92 & 0.22 & 2.23 \\
\hline F3 & 21.79 & 0.00 & $\mathbf{6 3 . 1 9}$ & 15.01 \\
\hline F4 & 30.20 & 0.04 & $\mathbf{6 0 . 6 4}$ & 9.12 \\
\hline Average & \multicolumn{5}{|c|}{$46.86 \%$} \\
\hline
\end{tabular}

Table 4.

PCA-HMM Based Fault diagnosis Confusion Matrix

\begin{tabular}{|c|c|c|c|c|}
\hline Label & F1 & F2 & F3 & F4 \\
\hline F1 & $\mathbf{5 4 . 0 7}$ & 38.34 & 1.71 & 5.89 \\
\hline F2 & 27.12 & $\mathbf{6 1 . 0 0}$ & 3.66 & 8.22 \\
\hline F3 & 3.33 & 1.30 & $\mathbf{5 9 . 4 8}$ & 35.89 \\
\hline F4 & 1.59 & 0.60 & 29.92 & $\mathbf{6 7 . 8 9}$ \\
\hline Average & \multicolumn{5}{|c}{$60.1 \%$} \\
\hline
\end{tabular}

Table 5.

Extended Segment Size for Original Data Euclidean Based Fault Diagnosis Confusion Matrix.

\begin{tabular}{|c|c|c|c|c|}
\hline Label & F1 & F2 & F3 & F4 \\
\hline F1 & $\mathbf{7 3 . 2 3}$ & 26.74 & 0.00 & 0.03 \\
\hline F2 & 13.49 & $\mathbf{8 6 . 5 1}$ & 0.00 & 0.00 \\
\hline F3 & 0.00 & 0.00 & $\mathbf{5 3 . 9 7}$ & 46.03 \\
\hline F4 & 0.79 & 0.09 & 19.93 & $\mathbf{7 9 . 1 9}$ \\
\hline Average & \multicolumn{5}{|c}{$73.22 \%$} \\
\hline
\end{tabular}

Table 6.

Extended Segment Size for Original Data PCA Based Fault Diagnosis Confusion Matrix.

\begin{tabular}{|c|c|c|c|c|}
\hline Label & F1 & F2 & F3 & F4 \\
\hline F1 & $\mathbf{7 0 . 7 9}$ & 15.01 & 0.11 & 14.10 \\
\hline F2 & 35.75 & $\mathbf{4 9 . 1 7}$ & 0.00 & 15.08 \\
\hline F3 & 30.53 & 0.00 & $\mathbf{5 3 . 9 7}$ & 15.50 \\
\hline F4 & 19.73 & 1.44 & 19.79 & $\mathbf{5 9 . 0 4}$ \\
\hline Average & \multicolumn{4}{|c}{$58.24 \%$} \\
\hline
\end{tabular}

The fifth experiment addresses the effect of increasing the segment size $\mathrm{K}$, where $\mathrm{K}$ is increased from 4 to 48 minutes. As expected, the recognition performance is enhanced after the 
segment size is increased. This is because the segment becomes more informative and discriminative. Table. 5 and Table. 6 illustrate the improvement in the recognition performance with respect to the corresponding results reported in Table.1 and Table.3, respectively.

\section{Conclusions}

We proposed a framework for automatic diagnosis of RAN faults. Experiments are conducted on data collected from live networks. The proposed framework achieved high diagnostic accuracy and can be easily implemented in an operator live network. It is worth to mention that although the proposed model was validated on diagnosing problems in the radio access of GSM networks only, the proposed method is also directly applicable in generic radio access networks (i.e. $3 \mathrm{G}$ Access Network) if the appropriate models are used. Since alarms as symptoms are normally available in the NMS of all operators, this makes the proposed automated tool easily to be implanted for different operators. A model has been built based on knowledge of GSM experts and databases from a real network to support the theoretical contributions of this paper.

Proposed tool can be easily extended to gather a lot of symptoms from different management systems to be used in the complex networks end to end fault diagnosis. That's drive down operational costs in the future cellular networks achieving fast fault recovery. Accordingly, it's expected to significantly improve the overall operational efficiency and enhance customer experience.

\section{REFERENCES}

[1] Hamalainen, Sanneck, Sartori. [2012] Self-Organising Networks (SON) Network Management Automation for Operational Efficiency. Wiley.

[2] Ciocarlie, G.F. , Chih-Chieh Cheng , Connolly, C. , Lindqvist, U. [2014] Demo: SONVer: SON Verification for Operational Cellular Network. Wireless Communications Systems (ISWCS), 2014 11th International Symposium on

[3] Tarmo Anttalainen. [2003] Introduction to Telecommunications Network engineering. 2nd Ed. Artech House telecommunications library

[4] Friedhelm Hillebrand. [2001] GSM and UMTS. John Wiley \& Sons Ltd

[5] Olasunkanmi F. Oseni, Segun I. Popoola, Henry Enumah, Ayonote Gordian. [2014] Radio Frequency Optimization of Mobile Networks in Abeokuta, Nigeria for Improved Quality of Service. International Journal of Research in Engineering and Technology,

[6] Asha K. Mehrotra. [2011] GSM System Engineering. Library of Congress Cataloguing in Publication Data.

[7] Halonen, T, J. Romero and J. Melero. [2002] GSM, GPRS and EDGE Performance Evolution towards 3G-UMTS. Wiley

[8] Baku, R., Nielsen, L., Guerrero, R., Hylander, G., \& Patel, S. [2002] Automated Troubleshooting of a Mobile Communication Network Using Bayesian Networks. In Proceedings of the IEEE International Workshop on Mobile and Wireless Communications Networks (MWCN'02). Stockholm, Sweden.

[9] Barco, R., Wille, V., Di'ez, L., \& La'zaro, P. [2006] Comparison of Probabilistic Models Used for Diagnosis in Cellular Networks. In Proceedings of the IEEE Vehicular Technology Conference VTC'06. Melbourne, Australia

[10] Raquel Barco, Volker Wille and Luis Diez. [2005] Special Issue on Self-organization in Mobile Networking System for Automated Diagnosis in Cellular Networks Based on Performance Indicator. European Transactions on Telecommunications Euro. Trains. Telecoms; 16:399-409. 
[11] Solana, B. ; Triola, J. ; Barco, R. ; Moltsen, L. ; Altman, Z. ; Lazaro, P [2008] Automated Diagnosis for UMTS Networks Using Bayesian Network Approach. IEEE Transactions on Vehicular Technology, Vol. 57, No. 4, July.

[12] L.R. Rabiner, and B.H. Jung [1986] an Introduction to HMMs, IEEE ASSP, Magazine, pp. 4-16, Janvier.

[13] Marc Moureta, b. Christine Solnona, b Christian Wolfa [2009] Classification of Images Based on Hidden Markov Models, Content-Based Multimedia Indexing. CBMI '09. Seventh International Workshop.

[14] C.-P. Liao and J. -T. Chen [2006], Maximum Confidence Hidden Markov Modelling, Department of Computer Science and Information Engineering, National Cheng Kung University, Tainan, Taiwan.

[15] M. El-Hanjouri, W.Alkhaldi, N.Hamdy, O.Abdel Alim [2002] Heart Diseases Diagnosis Using HMM, IEEE Melecon, pp. 489-491.

[16] Bing Wang, Tuan D. Pham, [2011] HMM-Based Brain Age Interpolation Using Kriging Estimator, Image and Signal Processing and Analysis (ISPA), 7th International Symposium, Sep.

[17] Lederman, D. Cohen, Arnon ; Zmora, Ehud ; Wermke, K. ; Hauschildt, S. ; StellzigEisenhauer, A. [2000] On the Use of Hidden Markov Models in Infants' Cry Classification., Electrical and Electronics Engineers.

[18] Li, Q.M., Zhao, T., Zhang, L., \& Lou, J. [2012]. , Mechanical Fault Diagnostics of on Load Tap Changer within Power Transformers Based on Hidden Markov Model, IEEE Transactions on Power Delivery, (Volume: 27, Issue: 2).

[19] Hasan Ocak Kenneth A. Loparo [2001] A New Bearing Fault Detection and Diagnosis Scheme Based on Hidden Markov Modeling of Vibration Signals, Acoustics, Speech, and Signal Processing, Proceedings. (ICASSP '01). IEEE International Conference (Volume: 5).

[20] Ajay R.Mishra [2004] Fundamentals of Cellular Network Planning and Optimisation - 2G 2.5G 3G Evolution to 4G, Wiley

[21] Lawrence R. Rabiner ,A tutorial on Hidden Markov Models and Selected Applications in Speech Recognition, Readings in Speech Recognition Pages 267-296

[22] L. Zhao and Y. Yang [1999], Theoretical Analysis of Illumination in PCA-Based Vision System, Pattern Recognition, Vol. 32, No. 4, PP. 547 -564

[23] Ozawa, S.; Ohta, R. [2011], Incremental Recursive Fisher Linear Discriminant for Online Feature Extraction, Evolving and Adaptive Intelligent Systems (EAIS), IEEE Workshop on, on page (s): $70-76$

[24] B. Kamo, S. Cakaj, V. Koliçi and E. Mulla [2012], Simulation and Measurements of VSWR for Microwave Communication Systems, Int'l J. of Communications, Network and System Sciences, Vol. 5 No. 11, pp. 767-773. Doi

[25] R. G. Vaughan and J. B. Andersen [1987], Antenna Diversity in Mobile Communications, IEEE Trans. Veh. Tech., vol. VT-36, pp.149-172 


\section{نظام تشخيص الأعطال الآلي للشبكات الخلوية على أساس نموذج ماركوف الكامن}

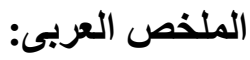

التشخيص الآلي و استكثناف الأخطاء و إصلاحها (TS) في شبكات النفاذ الر اديوي (RAN) للأنظمـة الخلويـة

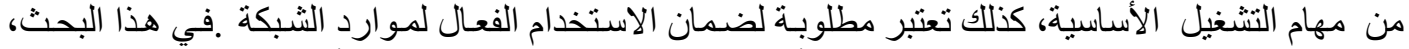

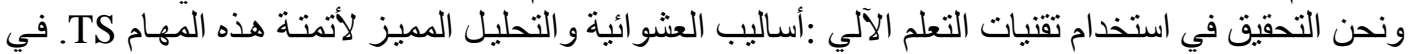

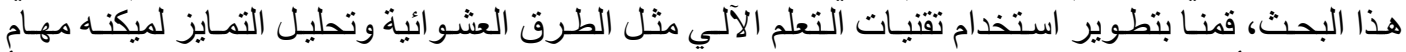

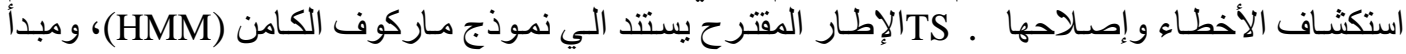

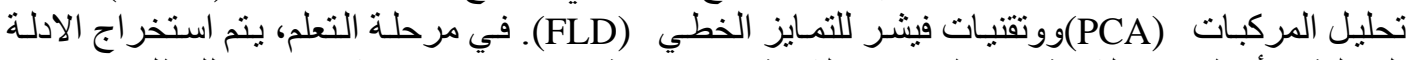

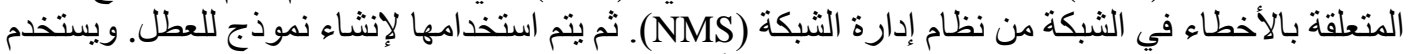

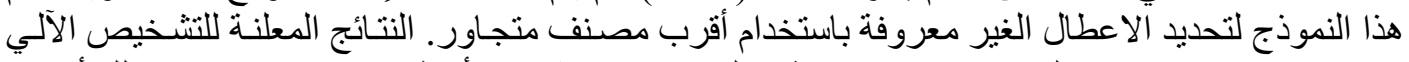

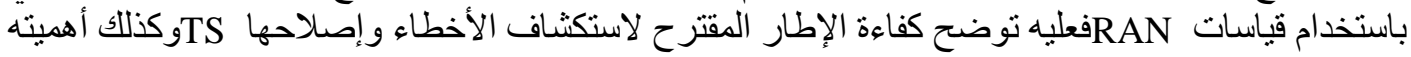
لمشغلي الثبكات المتنقلة. 